\title{
Linear beam raster for cryogenic targets
}

\author{
C. Yan*, N. Sinkine, R. Wojcik \\ Thomas Jefferson National Accelerator Facility, Physics Division, MS 12H, 12000 Jefferson Avenue, Newport News, VA 23606, USA
}

Received 11 May 2004; received in revised form 17 August 2004; accepted 2 September 2004

Available online 10 November 2004

\begin{abstract}
Based on the H-bridge switch technique a linear beam raster system was developed in 2002. The system generates a rectangular raster pattern with highly uniform $(\sim 95 \%)$ raster density distribution on cryogenic targets. The two raster frequencies are 24.96 and $25.08 \mathrm{kHz}$. The turning time at the vertex is $200 \mathrm{~ns}$ and the scan linearity is $98 \%$. The beamheating effect on the target is effectively eliminated. The new raster system allows the use of higher beam current toward $200 \mu \mathrm{A}$ in many of the experimental proposals at end station Hall A and Hall C of the Jefferson lab.
\end{abstract}

(C) 2004 Elsevier B.V. All rights reserved.

PACS: 29.27.-a; 41.25.Fr

Keywords: Beam raster; Cryogemic targets; Beam heating; H-bridge

\section{Introduction}

At the Jefferson lab, the Lissajous beam raster system has been used for cryogenic target experiments in two end stations, Hall A and Hall C, since 1996 [1]. The major part of the system is the high $Q$ resonance loop, which consists of the aircore raster magnet, resonance capacitors and power audio-amplifier. The waveform of the magnet current is sinusoidal. As the sinusoidal waveform approaches its peak, it slows down in order to reverse direction at the edge of the scan

\footnotetext{
*Corresponding author. Tel.: +17572697349; fax: +17572697363 .

E-mail address: yan@jlab.org (C. Yan).
}

region. At this location, the scanning velocity of the electron beam becomes nearly zero. This causes much more beam energy to be deposited along the boundaries and at the four corners as shown in raster density two-dimensional (2D) and three-dimensional (3D) histograms of Figs. 1 and 2 .

This large increase in the deposit beam energy in this area eventually causes overheating of the target material. By the experimental measurement, a luminosity scan with either High-Resolution Spectrometer (HRS) in Hall A or High Momentum Spectrometer (HMS) in Hall C, we found that the luminosity decreases gradually with an increase in the beam current. It indicates that the local overheating effect near the boundaries and the 

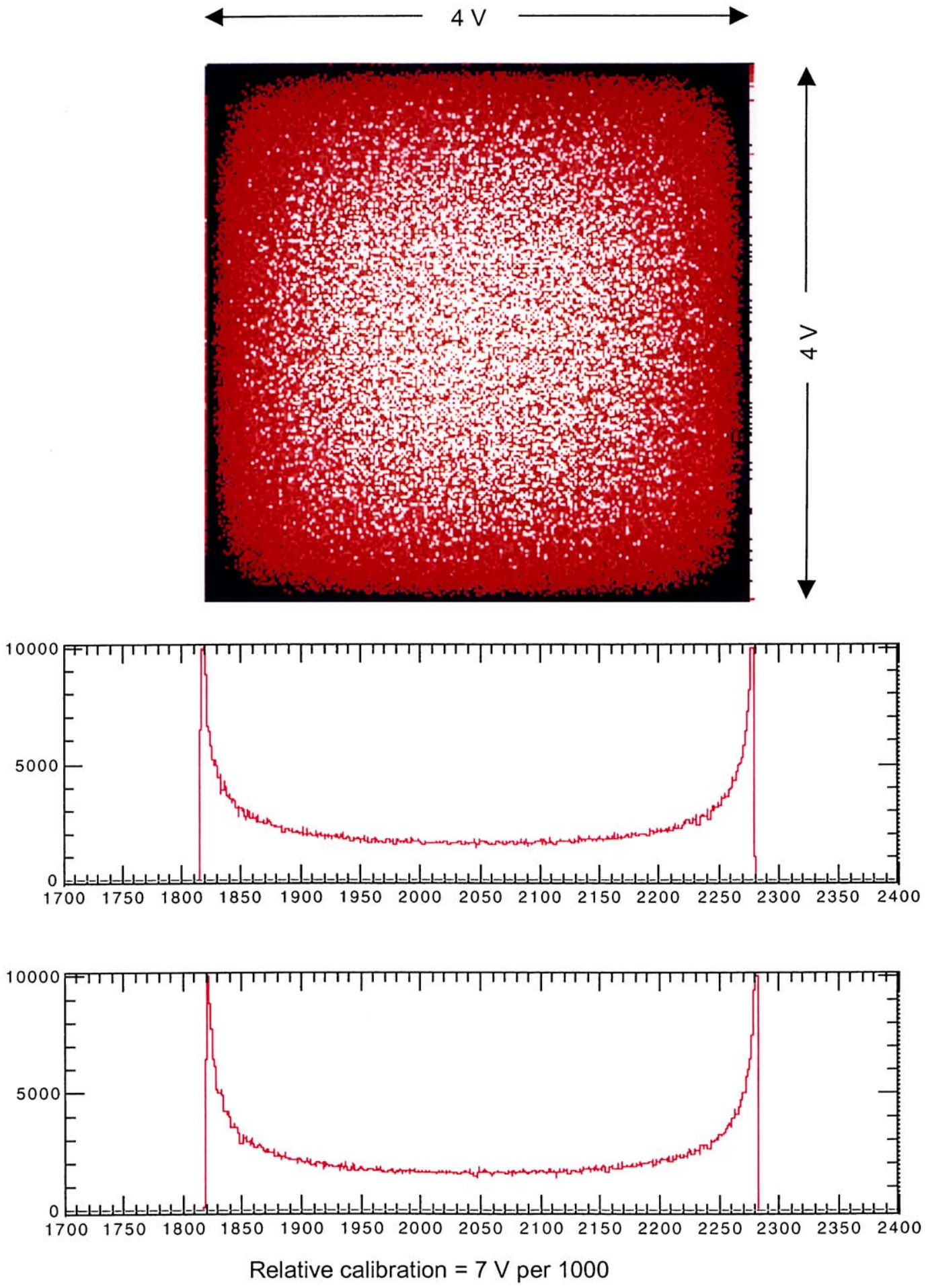

Fig. 1. The 2D histogram of magnet current signals from the Lissajous target raster system (1996-2002). 


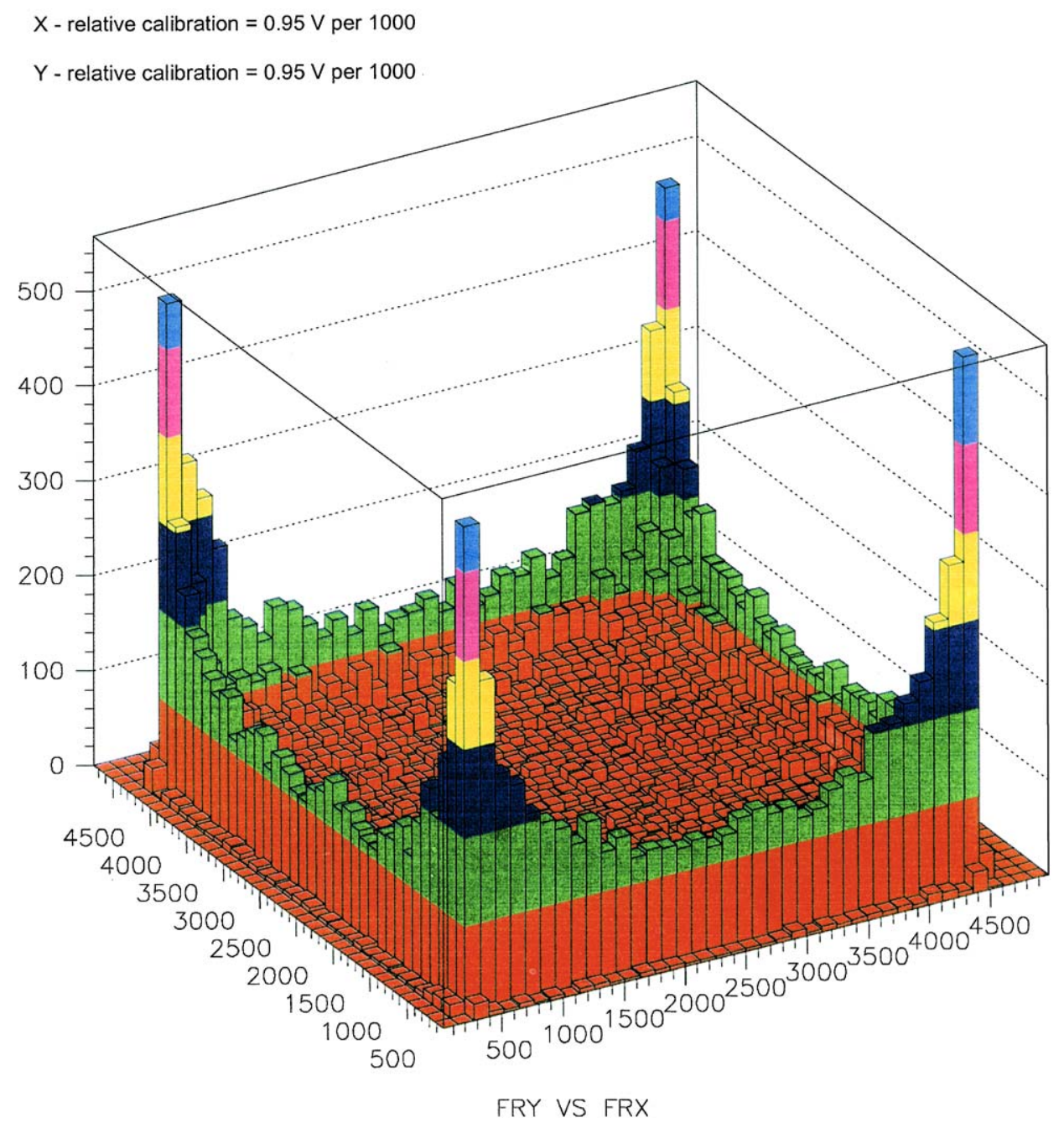

Fig. 2. The 3D histogram of magnet current signals from the Lissajous target raster system (1996-2002) obtained by using Hall C data aquisiton system. At the corners, the density can be 10 times higher than the central region.

corners of the Lissajous raster pattern contributes an uncertainty in target length, which in turn affects the accuracy of the experimental data.

The first linear raster system has been operating since August 2002. With the use of this newly developed system, the estimated maximum allowable beam current without target boiling is about $200 \mu \mathrm{A}$. The design of this new system focused on two points - to create highest linear velocity and to suppress the turning time as much as possible. Compared with the Lissajous raster, the new system provides a highly homogeneous raster density distribution over the entire raster region with $98 \%$ linearity, $95 \%$ uniformity, and $1000 \mathrm{~m} / \mathrm{s}$ linear sweep velocity. The turning time at the vertex of the raster pattern is about $200 \mathrm{~ns}$ while the beam traveling time from edge to edge is $20 \mu \mathrm{s}$; therefore, beam dwelling is almost negligible. The $2 \mathrm{D}$ raster density profile of the linear raster is shown in Fig. 3 and Fig. 4 is the 3D histogram. 

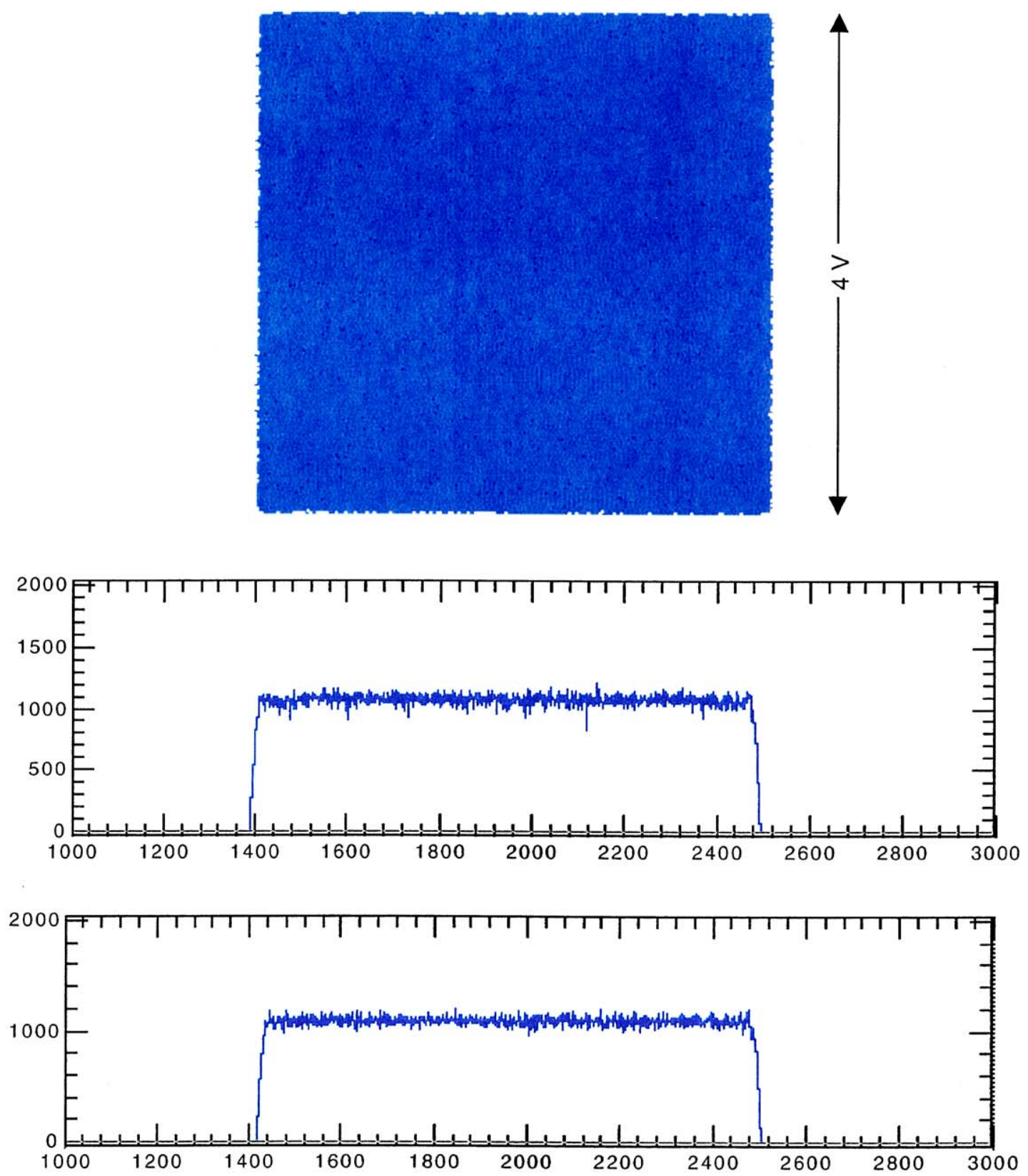

relative calibration $=3.7 \mathrm{~V}$ per 1000

Fig. 3. The $2 \mathrm{D}$ histogram of magnet current signals from the linear raster system. 


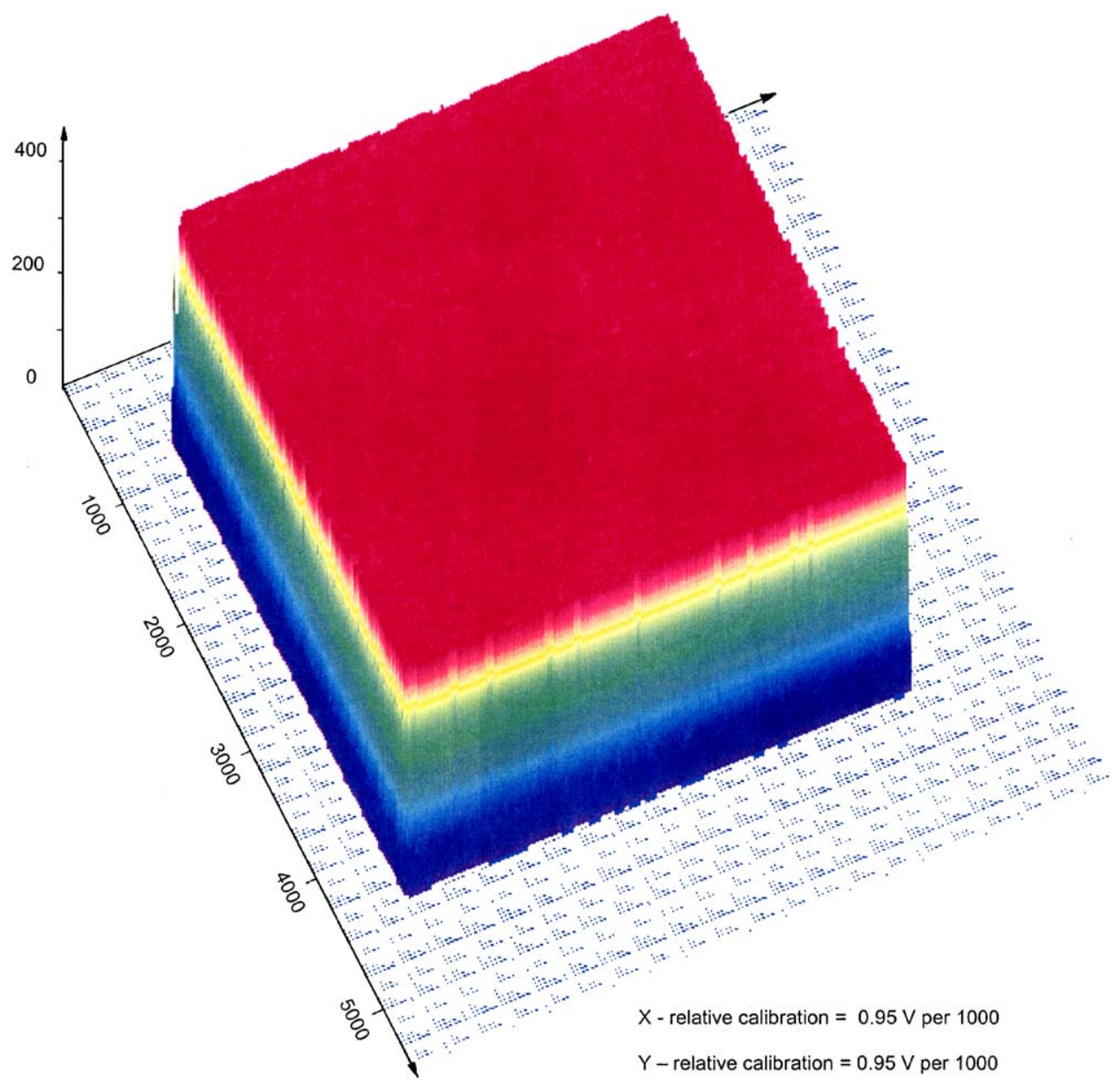

Fig. 4. The 3D histogram of magnet current signals from the linear raster system.

\section{Heating effect in the cryogenic targets}

When an electron beam passes through a cryogenic target, heat is generated mainly through the ionization energy loss, which can be calculated using the theory of Bethe [2] and Bloch [3,4]. In a cryogenic target, most of the bremsstrahlung photons escape from the target without being absorbed. They contribute relatively little to the increase in target temperature. Thermal energy produced in the cryogenic target is transferred by the liquid material flow through the target cell, cooling loop, and heat exchanger.

A simplified adiabatic model [5] is used for a quantitative estimation of the heating effect in the cryogenic target: the material flow between the target cell and reservoir is assumed as the only dominant factor and other heat transfer processes, such as transverse convection, thermal conduction through the cell boundaries, and radiative cooling, are eliminated in consideration.

\subsection{Local heating}

The local heating concept describes instantaneous status under adiabatic approximation-the beam deposits energy along its trajectory and the heat produced by the beam power has not been spread all over the volume of the target cell. Local heating occurs before the heat distribution reaches 
equilibrium in the entire volume of the target cell. For a new raster system design, the local heating effect is the most important factor of consideration of physical constraints.

If the beam is well focused to a small dimension along the beam trajectories, the liquid hydrogen is overheated by the deposit beam energy. In consequence, bubbles will be formed along the path. Target density variation is eventually generated.

The temperature rise by local heating can be calculated by the formula below:

$\Delta T(\mathrm{~K})=\frac{Q(\mathrm{~J})}{M(\mathrm{~g}) C_{p}(\mathrm{~J} /(\mathrm{gK}))}$

where $Q=\Delta P \cdot \Delta t_{\mathrm{s}}$ is the total deposit energy by the electron beam along the trajectory. The beam deposit power $\Delta P=\rho\left(\mathrm{g} / \mathrm{cm}^{3}\right) \mathrm{d} E / \mathrm{d} x(\mathrm{MeV} /$ $\left.\left(\mathrm{g} / \mathrm{cm}^{2}\right)\right) L(\mathrm{~cm}) I(\mu \mathrm{A}) . \quad C_{p}=10.38 \mathrm{~J} /(\mathrm{g} \mathrm{K})$ is the specific heat of liquid hydrogen. $M$ is the total mass of target liquid involved in the active volume. $\Delta t_{\mathrm{s}}$ is the beam interaction time with the target fluid.

The total beam deposit energy during $\Delta t_{\mathrm{s}}$ can be expressed as

$$
\begin{aligned}
Q(\mathrm{~J})= & \rho\left(\mathrm{g} / \mathrm{cm}^{3}\right) \mathrm{d} E / \mathrm{d} x\left(\mathrm{MeV} /\left(\mathrm{g} / \mathrm{cm}^{2}\right)\right) \\
& \times L(\mathrm{~cm}) I(\mu \mathrm{A}) \Delta t_{\mathrm{s}}
\end{aligned}
$$

where $\mathrm{d} E / \mathrm{d} x=5.2 \mathrm{MeV} /\left(\mathrm{g} / \mathrm{cm}^{2}\right)$ is the electron energy loss in liquid hydrogen. For Hall $\mathrm{C}$ LH targets, the direction of stream velocity $v_{\mathrm{s}}$ is perpendicular to the beam; so $\Delta t_{\mathrm{s}}=d / v_{\mathrm{s}}$. Here, $d=0.01 \mathrm{~cm}$ is the beam transverse dimension. $M$ is the mass of the target material inside the trajectory boundary. $M(\mathrm{~g})=$ $d^{2}\left(\mathrm{~cm}^{2}\right) L(\mathrm{~cm}) \rho\left(\mathrm{g} / \mathrm{cm}^{3}\right), \quad \rho=0.07 \mathrm{~g} / \mathrm{cm}^{3}$ is the density of liquid hydrogen, $L=15 \mathrm{~cm}$ is the geometric length of the target cell.

Finally, we have

$\Delta T(\mathrm{~K})=\frac{\mathrm{d} E / \mathrm{d} x\left(\mathrm{MeV} /\left(\mathrm{g} / \mathrm{cm}^{2}\right)\right) I(\mu \mathrm{A})}{d(\mathrm{~cm}) C_{p}(\mathrm{~J} /(\mathrm{gK})) v_{\mathrm{s}}(\mathrm{cm} / \mathrm{s})}$.

For Hall C 4 and $15 \mathrm{~cm}$ target cells, the stream velocity is about $100 \mathrm{~cm} / \mathrm{s}$. At $200 \mu \mathrm{A}$ beam current, the local temperature rise is about $100 \mathrm{~K}$. Usually, the target is subcooled by $2 \mathrm{~K}$; that means the target material can only be warmed up to $2^{\circ}$ before reaching the vapor pressure curve appropriate to its operating pressure. The allowable temperature rise along the beam path can be set at only $1-2^{\circ}$. If $1^{\circ}$ is applied, the local heating causes excessive temperature rise by a factor of 100 .

\subsection{Strengthen the target endurance}

Two options could be used to increase allowable beam current. One is to increase the transverse dimension of the beam, another is to increase the stream velocity of the circulation of the cryogenic liquid material.

The effective transverse beam dimensions can be increased by defocusing the beam. But it is not the perfect solution because the intrinsic beam structure will be magnified and shown on the target as the density fluctuation of the primary beam, and a static large beam spot on the target may not match the spectrometer resolution. The quadrupoles will generate a large incident angle when strong defocusing is requested.

The stream velocity of the target cryogenic liquid is limited by the frequency of the turbo fan in the circulation loop of cryogenic liquid. In general, the fan frequency is below $100 \mathrm{~Hz}$ and is confined by several factors, such as maximum torque of the motor, shape of the blade, electric smoothness of the motor drivers, and the style of the motors. As a matter of fact, the effective (or equivalent) stream velocity can be greatly increased by applying the beam raster to move the beam very fast away from its original trajectory. The function of increasing the stream velocity of the target fluid and the function of increasing the linear velocity of the beam raster are completely equivalent.

Let us consider 1D approximation and take the previous calculation result of the local heating effect as an example. In order to keep the local temperature rise below $1^{\circ}$, the equivalent stream velocity must be increased by a factor of 100 . Assume the raster amplitude $d_{\text {raster }}$ is $0.2 \mathrm{~cm}$, raster track is absolutely linear, stream velocity is $100 \mathrm{~cm} / \mathrm{s}$. The required raster frequency for a linear raster system can be calculated from the following formula:

$f_{\text {raster }}=100 \times V_{\mathrm{s}}(\mathrm{cm} / \mathrm{s}) / 2 d_{\text {raster }}(\mathrm{cm}) \sim 25 \mathrm{kHz}$. 
The figure $25 \mathrm{kHz}$ was applied as the basic constraint of the linear raster system design.

In fact, this is a very conservative estimation because the target operates in a complete turbulent flow regime. The Reynolds number of Hall C LH flow is about 40,000. A fast material exchange occurs inside the target cell. The convection and transfer processes along the beam path boundary become indelible.

\section{Raster magnets}

\subsection{Location of target raster systems}

The magnets of the target raster are installed on a non-metallic (G-10 material) girder. In Hall C, the locations of the two magnets are about $20 \mathrm{~m}$ upstream from the center of the target chamber with no optical focusing elements between. The raster magnets are in free-drift space in order to keep the independence of the raster operation/ calibration to the beam optics tuning. In Hall A, the target rasters are located upstream the Compton polarimeter and four beam line quadrupoles. The final raster size on the target will be determined by the combination of the magnet current reading and optics matrix elements.

\subsection{Air-core magnets}

Bedstead air-core windings made of Litz cables are used to construct target raster magnets. Compared with the iron-core magnet, air-core magnets can be operated at relatively higher frequencies with low-field integral value for applications of small bending power. No hysteresis exists in the air-core coil. The response between the excitation current and the induced magnetic field is spontaneous. The field information can be accurately obtained from either the driving current sensor or the pickup coil on the magnet.

The penetration depth in a copper conductor can be calculated from formula $\delta=65.5 /\left(f_{0}\right)^{1 / 2}$. At $25 \mathrm{kHz}, \delta \sim 0.42 \mathrm{~mm}$. There is a special consideration of the strand size of magnet windings.

When the conductor carries an alternating current, which is the case of raster magnets, the conductor is in an alternating magnetic field, which will generate EMFs in the conductor resulting in eddy currents and associated power losses. The magnitude of the eddy current (resistance increase) becomes larger as frequency and wire diameter increase. To obtain satisfactory results, a mere subdivision of a solid wire into a number of strands is not sufficient.

Special "Litz" cables were used as magnet wire for production of Jlab raster magnets. Litz is a short term used for a German word "Litzendraht" that means braided wire. Litz cable consists of multiple strands and each strand is coated by insulation film. The entire cable is also subdivided into several twisted groups, which are formed by twisted basic strands. The major purpose for use of the Litz cable is to reduce power loss caused by the Eddy current.

The linear raster system operates at a $25 \mathrm{kHz}$ triangular current wave form. At least, the third harmonic $(75 \mathrm{kHz})$ should be reserved. So the target raster FR magnet winding is made of 1650 strands Awg 38 Litz wire $(5 \times 5 \times 66)$, which can be operated at a frequency range from 50 to $100 \mathrm{kHz}$.

The bedstead coil is the most effective geometry because the magnet apertures fit the beam pipe diameter and it has a moderate uniform field volume. The experimental parameters of the FR magnets are listed in Table 1.

\subsection{Raster optics}

Under Sharp Cut Off Fringing Field (SCOFF) approximation, the magnetic field of the raster magnet is considered as a container with total length $L_{\text {eff }}$ (effective length) and a uniform dipole field everywhere inside, but zero field outside. The total field integral is equal to the product of the effective length and the central field value. The deflection angle can be calculated from the classic formula

$B \rho=33.356 p$

where $B$ is the magnetic field intensity in $\mathrm{kG}, \rho$ the deflection radius in $\mathrm{m}$, and $p$ the particle momentum in $\mathrm{GeV} / c$. At a small deflection angle, e.g., the FR deflection angle is usually $0.05 \mathrm{mrad}, \phi \approx$ 
Table 1

The major experimental parameters of raster magnet

\begin{tabular}{ll}
\hline Magnet ID & Targer raster magnet \\
Central field at $1 \mathrm{a}(\mathrm{G})$ & 3.41 \\
Max. bending power at $12 \mathrm{GeV} / c$ & 0.08 \\
(mrad) & 81 \\
Field integral $(\mathrm{Gcm} / \mathrm{A})$ & 23.7 \\
Effective length $(\mathrm{cm})$ & 25 \\
Longitudinal dimensional $(\mathrm{cm})$ & 1.905 \\
Inner radius of the gap $(\mathrm{cm})$ & 12 \\
Number of terms & 1200 \\
Operational ampere-turns $(\mathrm{A}-\mathrm{T})$ & 1.0 \\
RMS current density $\left(\mathrm{A} / \mathrm{mm}^{2}\right)$ & 88 \\
Inductance $(\mu \mathrm{H})$ & 0.04 \\
DC resistance $(\Omega)$ & $10^{-2}$ \\
Field uniformity $(\Delta B / B)$ & $2 \times 2 \times 20$ \\
Uniform field volume $\left(\mathrm{cm}^{3}\right)$ & $30 \times 30 \times 40$ \\
Stray field volume $(<1 \mathrm{G})\left(\mathrm{cm}^{3}\right)$ & Litz $1650($ Strand \\
Type of conductors & Awge 38$)$ \\
& 120 \\
Quality factor of windings & $25($ triangle) \\
Operational frequency $(\mathrm{kHz})$ & 10 \\
Weight $(\mathrm{lb})$ &
\end{tabular}

$\sin \phi \approx \tan \phi$. The deflection angle in the unit of radian can be written as

$\phi=\frac{\int B \mathrm{~d} l}{33.356 p}$.

The raster amplitude is equal to

$\delta x_{1 / 2} \sim L \phi=L \times \frac{\int B \mathrm{~d} l}{33.356 p}$

where $L$ is the distance from the center of the raster magnet to the target.

\subsection{Raster magnet mapping}

The field integral data of raster magnets are extracted from DC mapping data provided by the Jlab magnet group [6]. Identical measurements were made for both vertical and horizontal magnets. All measurements were made at $10 \mathrm{~A}$ DC magnet current. Data were collected using the Hall Probe Stepping Stand in the Magnet Measurement Group. A Group-3 Hall probe was translated longitudinally through the magnet between $\pm 27.5 \mathrm{~cm}$ from the longitudinal center. The Hall probe was read in $0.5 \mathrm{~cm}$ steps over this span. Longitudinal data sets were collected at three transverse locations in the magnet $(x=0.0,+0.5$ and $-0.5 \mathrm{~cm})$. The longitudinal field profile is shown in Fig. 5.

In the data analysis a constant offset of $0.24 \mathrm{G}$ was subtracted from all data and integrals calculated. The offset is the sum of the terrestrial field and Hall probe zeroing offset. A value of 0.24 was determined from the average of all readings at magnet ends where the field was constant. The average effective length is about $23.7 \mathrm{~cm}$. The average field integral per amp is $81 \mathrm{Gcm}$ and central field is $3.41 \mathrm{G} / \mathrm{A}$.

When the field integral data from DC mapping is used for raster bending angle calculation, there is $\sim 1 \%$ slight deficiency of $\int B \mathrm{~d} l$ of DC mapping data because of the attenuation of alternative field at the end flanges of the ceramic beam pipe at $25 \mathrm{kHz}$. From the correlation of the field integral and the excitation current, one can finalize the setting formula by the magnetic current. As a cross check, by scanning the two wire scanners [6] in front of the target, the rastered beam size can be obtained from beam profiles. The optics-setting parameters should be slightly corrected by the beam-profile data [7].

\section{H-bridge power switch}

As shown in Fig. 6 the principle of H-bridge is to apply a bipolar switching high voltage through a $2 \times 2$ diagonal switch to a floating raster air-core magnet, which is an inductive load with low losses. When the two sets of diagonal switches are synchronized to be turned on and off, the current waveform in the magnet will follow an exponential rise and decay curves. If the period of switching waveform is much shorter than the time constant, the current waveform is approximately linear. Fig. 7 shows the output current waveform from the Pearson 411 current sensor, referring to the TTL synchronization outputs.

\subsection{Estimation of major performances}

\subsubsection{H-bridge circuit analysis}

The H-bridge consists of two diagonal switches and a floating inductive load. Let us take a simple 


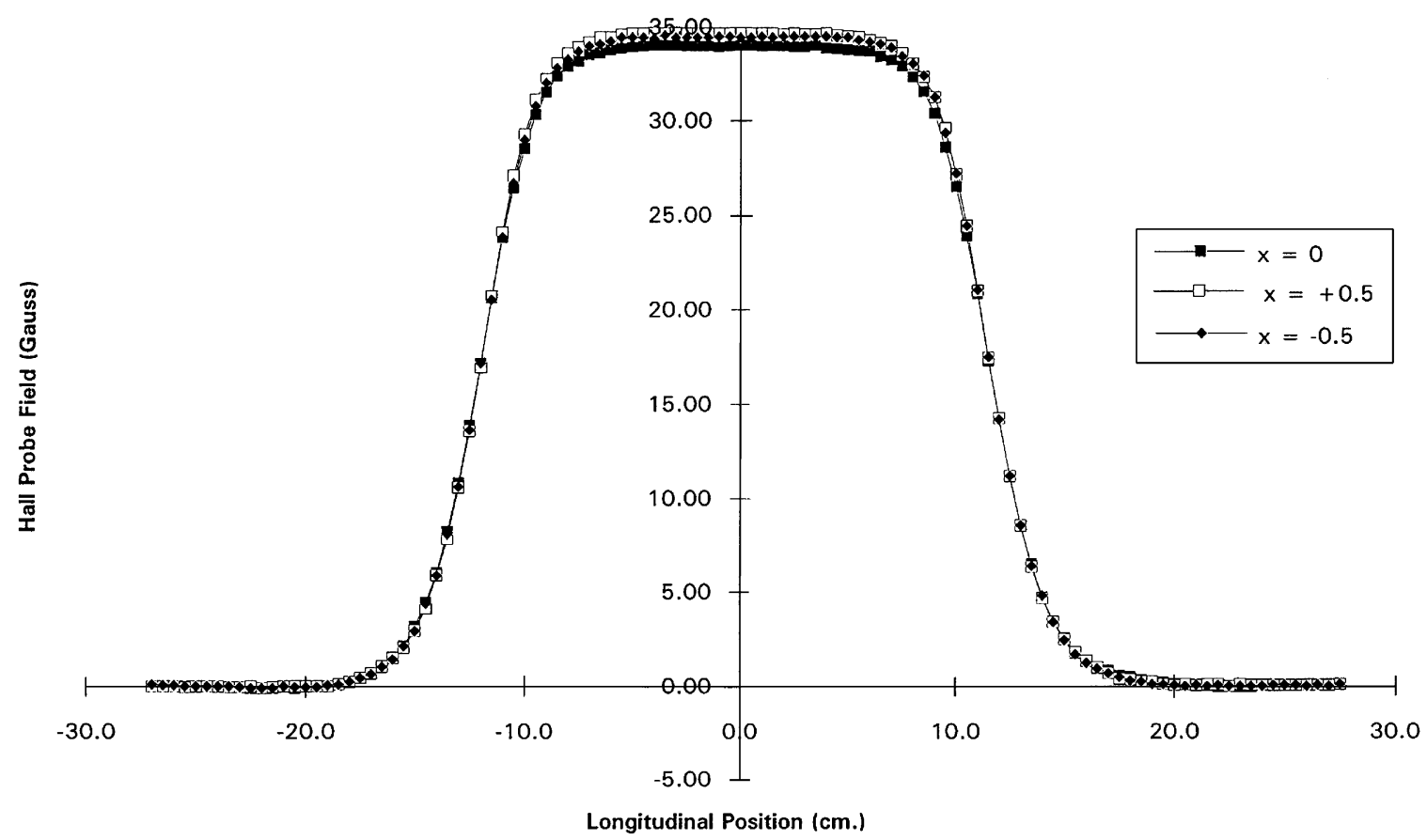

Fig. 5. The longitudinal field profiles of the raster magnet at three transverse locations: $x=0$ and $\pm 0.5 \mathrm{~cm}$ from DC mapping data.

model to find the basic switching performance of an H-bridge as shown in Fig. 6. The load consists of inductance $L$ and resistance $R$. Apply Kirchhoff's loop equation:

$\varepsilon-I R-L \frac{\mathrm{d} I}{\mathrm{~d} t}=0$.

We have a solution:

$I(t)=\frac{\varepsilon}{R}\left(1-\mathrm{e}^{-(R / L) t}\right)=\frac{\varepsilon}{R}\left(1-\mathrm{e}^{-t / \tau}\right)$,

where the time constant $\tau=L / R$.

In one diagonal path, $R=2 R_{1}+R_{2}, R_{1}$ is the 'ON' resistance of the HEXFET switch (FA57SA50LC, $R_{\mathrm{DS}(\text { on })}=0.08 \Omega$ ), and $R_{2}$ is the DC resistance of the magnet. Because two HEXFETs are connected in parallel, we have $R_{1}=0.04 \Omega$, $R_{2}=0.04 \Omega, \quad R=2 R_{1}+R_{2}=0.12 \Omega, \quad L=88 \mu \mathrm{H}$ and we have $\tau=0.73 \mathrm{~ms}$. The half of raster period $T_{1 / 2}=20 \mu \mathrm{s} \ll \tau$. Now the scan trace trajectory is approximately linear because

$I(t)=\frac{\varepsilon}{R}\left(1-\mathrm{e}^{-(R / L) t}\right) \approx \frac{\varepsilon}{R}$.
The non-linearity $=1-(I(0)-I(0.5 T)) / I(0)=$ $1-\mathrm{e}^{-T / 2 \tau}=0.027$.

\subsubsection{Switching performance of $\mathrm{H}$-bridge}

The clarity of the boundaries of the raster pattern indicates how fast the beam changes direction at the vertex. It is determined by the dwell time of the rastered beam near the peak. Both the switching performance of diagonal switch elements and beam size are involved.

The sum of the switching delay time and rise/fall time of the HEXFET FA57SA50LC element is about $200 \mathrm{~ns}$. The switching performance of H-bridge is shown in Fig. 8- the photo of both current and sync waveforms at the turning point. The normal beam size is $100 \mu$. At $25 \mathrm{kHz}$ raster frequency, if we take the half as the resolving measure, the dwelling time due to beam size effect is about $2 \mu \mathrm{s}$. The switching time of $\mathrm{H}$-bridge is 10 times shorter than the dwell time of the beam size effect. Therefore, the contribution of H-bridge switching to the dwelling time is negligible. 

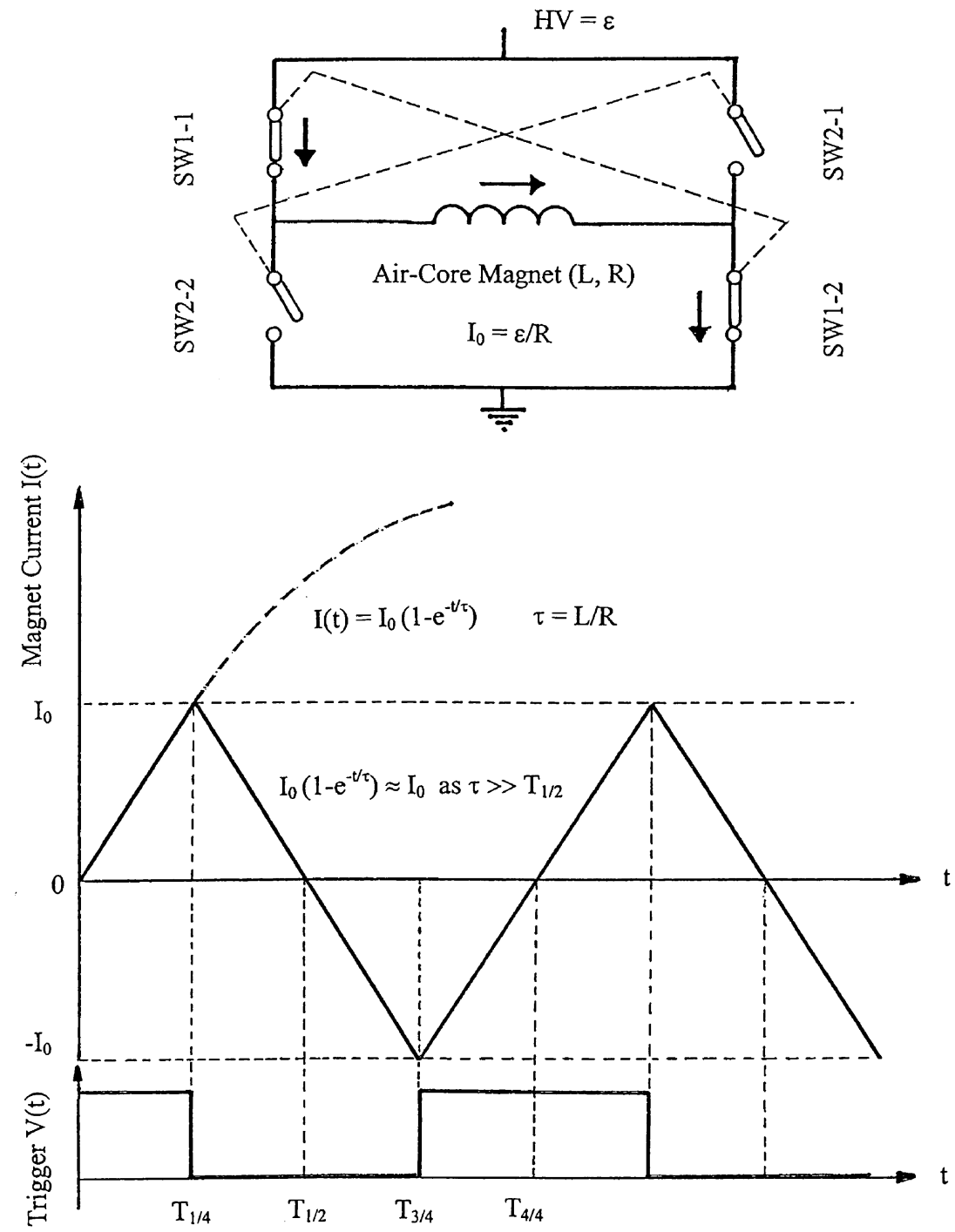

Fig. 6. The operation principle of the H-bridge switch. The upper picture is a simplified diagram of the $2 \times 2$ switch and a pure inductive load is connected to the centers of the high and low shoulders. The lower part shows the current waveform under a periodic switching.

\subsubsection{Voltage of single polarity power supply}

When the switches are 'ON', $\varepsilon=V_{0}=$ applied voltage, we have

$V_{0}=I R+L \frac{\Delta I}{\Delta t}$

where $\quad R=2 R_{1}+R_{2}=0.12 \Omega, \quad I R=6 \mathrm{~V}, L=$ $88 \mu \mathrm{H}, \Delta I_{\max }=50 \mathrm{~A}, \Delta t=0.5, T=20 \mu$ s and we have $V_{0} \sim 446 \mathrm{~V}$.
UltraVolt $\frac{1}{2} 24-\mathrm{P} 250 \quad(500 \mathrm{~V}$ adjustable $250 \mathrm{~W}$ DC-to-DC converter) is used to power H-bridge. Due to the simplicity of equation

$$
V_{0}=I R+L \frac{\mathrm{d} I}{\mathrm{~d} t}
$$

a linear relation between raster current and applied voltage is straightforward at a fixed 


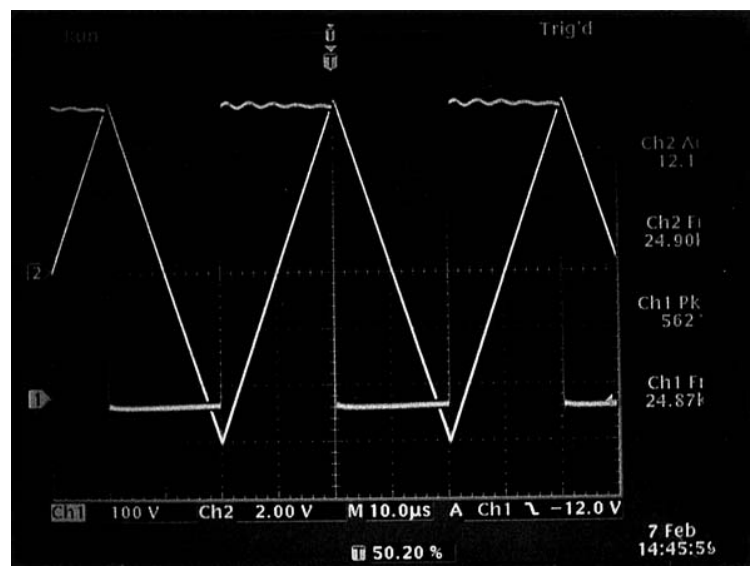

Fig. 7. The output current waveform of H-Bridge from the Pearson current sensor referring to the synchronization signals.

switching frequency ( $T$ is a constant). The slope of $V_{\max }-I_{\max }$ line is determined by $R+2 L / T$.

\subsubsection{Energy storage capacitors}

In order to reduce the capability requirement to the DC power supply, energy storage capacitors are used to boost the low-power output power supply $(250 \mathrm{~W})$. During half of the raster period, the voltage swing is $50 \mathrm{~V}$. The energy stored in the capacitor is

$E_{1}=\frac{1}{2} C V^{2}$

and dissipate energy through the $\mathrm{H}$-bridge is

$E_{2}=\frac{1}{2} L I^{2}+I^{2} R \frac{1}{2} T$.

To make $E_{1} \gg E_{2}$, four $165 \mu \mathrm{F} 600 \mathrm{~V}$ capacitors in parallel are sufficient to maintain stable voltage over H-bridge during a continuous raster operation.

\subsection{Configuration of $\mathrm{H}$-bridge}

Fig. 9 is a photo of a complete $\mathrm{H}$-bridge configuration on the heat sink. The eight HEXFETs are installed on the heat sink. On the photo the view of them is partly blocked by the bus strips, snubber capacitors, and the driver boards. All the components of the $\mathrm{H}$-bridge are arranged in such a way that the power leads of the drain and
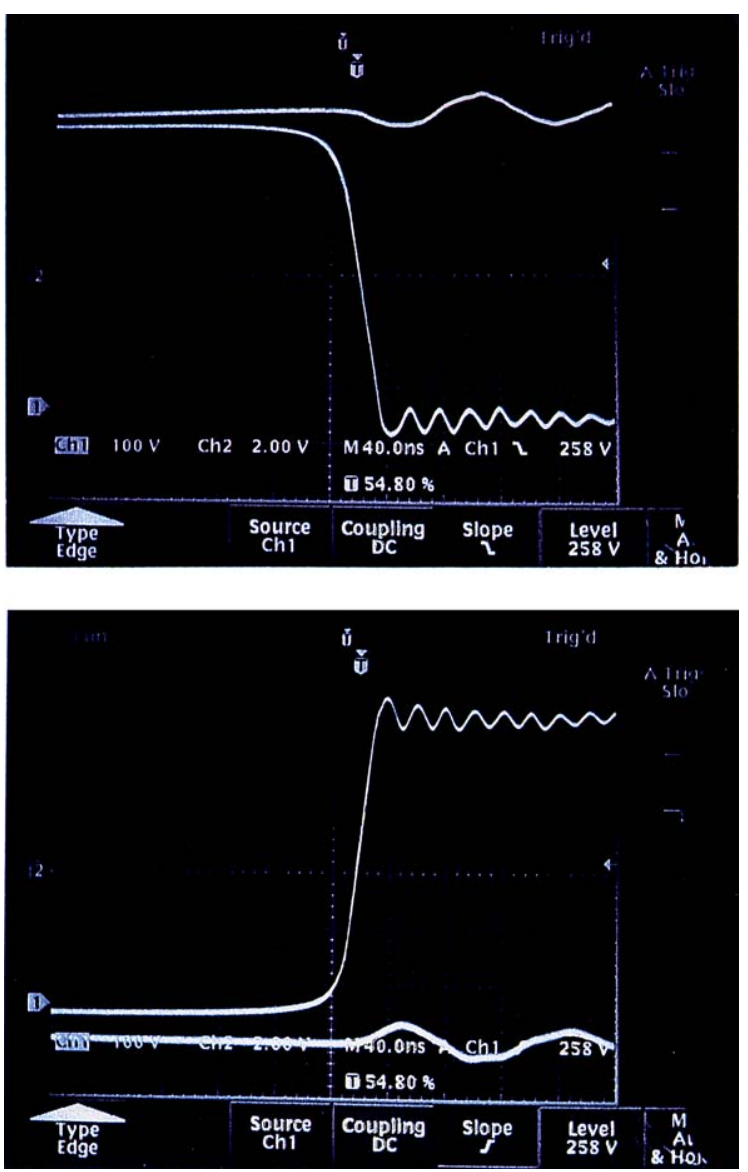

Fig. 8. The detailed view of the magnet current waveform near the turning point from the Pearson current probe. Under extension of time base, the vetex of triangular current waveform shows three ripples. The switching time is about $200 \mathrm{~ns}$. Upper and lower photos indicate up $\rightarrow$ down and down $\rightarrow$ up, the two types of vertices, respectively.

source of HEXFETs are connected by the shortest silver-plated copper bus strips with $4 \mathrm{~mm}$ thickness. The snubber capacitors are directly connected on HEXFET modules. The four HEXFET driver boards are also directly loaded on the HEXFET module without any wire connections. The power terminals of the H-bridge output, the leads of the magnet, the input terminals of $\mathrm{HV}$ power supply, and the terminals of storage capacitors are connected in the same way. This configuration effectively eliminates 


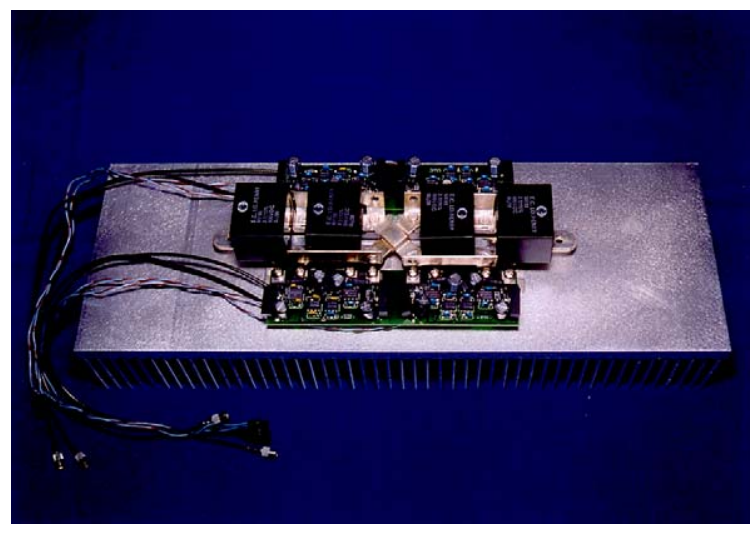

Fig. 9. The configuration of H-bridge on the heat sink. The major switch elements MOSFETs are directly connected by silver-plated copper bus strips and snubber capacitors are also directly located on the HEXFETs.

parasitic inductance between the bridge elements, and thereby suppresses spikes caused by switching HV-powered HEXFETs.

Each HEXFET is driven by a single channel of the MOS HEXFET driver MAX4429 through optocoupler HCPL7710. They are powered by a floating-supply voltage generated from the Polytron SWU 0.75-5S15 DC/DC converter.

\subsection{Raster frequencies}

As mentioned in the previous section, in order to eliminate local heating in the full range of the beam current, the fundamental frequency of the new linear raster system should be about $25 \mathrm{kHz}$. We also want the beam spot traveling as long a distance as possible. So far, we selected a scanning mode that has a single diagonal scan line in one pattern frame. Two frequencies with different ratio were tested by Lissajous pattern display on the scope. The best ratio was found as 1.00481 because of the following

1. The pattern is quickly rolling, we may expect to have the largest raster area coverage per time unit.

2. The pattern is stable to frequency variations.

3. The two frequencies stand far away from formation of the standing wave.
4. The two raster frequencies must be common multiples of beam spin reversal frequency for the further application of the phase lock.

Inside the power module, raster frequency is locally generated from the crystal oscillator. When no input of external helicity frequency presents, the module turns automatically to its built-in raster frequency in free-run mode.

\subsection{Raster frequency synchronization}

Experiments with polarized beam, e.g. the $G_{0}$ experiment, require that the raster pattern (instant beam positions in both $x$ and $y$ directions) is exactly correlated with the beam spin reversal frequency, i.e. helicity frequency. A special electronics of raster frequency generation was designed to serve this task.

The block diagram of raster frequency generation is illustrated in Fig. 10. The 74HCT4046A PLL chip generates $5.21664 \mathrm{MHz}$ from input helicity frequency. The $5.21664 \mathrm{MHz}$ output frequency is a common multiple of the two raster frequencies $(25.08 \mathrm{kHz} \times 208$ and $24.96 \mathrm{kHz} \times$ 209). The two synchronized raster frequencies are logically-subtracted to produce $120 \mathrm{~Hz}(25.08-$ $24.96 \mathrm{kHz}=120 \mathrm{~Hz}$ ). After dividing by 4 the $30 \mathrm{~Hz}$ frequency signal is sent back to 4046A PLL as PLL-feedback input. As the final output, the synchronization circuit always generates the two raster frequencies as $832 f_{\text {helicity }}$ and $836 f_{\text {helicity. The }}$ two raster systems start ramping up at exactly the same time from the leading edge of helicity signals.

The phase-lock accuracy is determined by phase jitters of the PLL circuit. It is in the order of $10^{-5}$

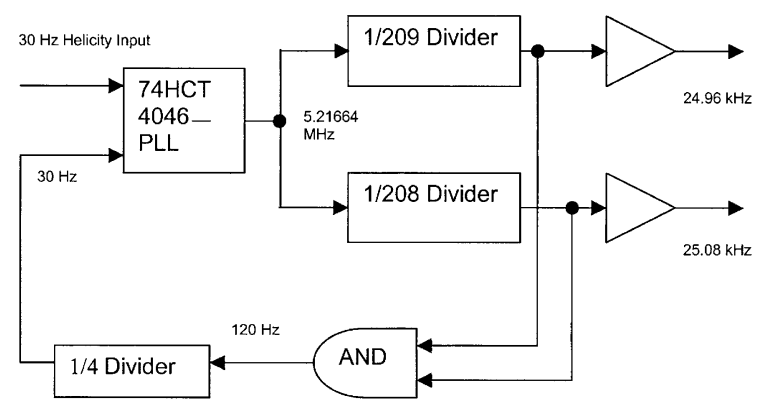

Fig. 10. The conceptual diagram of raster frequency generation from helicity synchronization. 
of input frequency. The PLL jitter is eventually transferred to $25 \mathrm{kHz}$ raster frequency output showing $1 \mu$ s phase fluctuation.

\section{The overall performance and experimental application}

The major operational parameters of the linear raster system from experimental tests are listed in Table 2.

The first linear target raster system was installed in Hall $\mathrm{C}$ to replace the original Lissajous raster in August 2002. The second system was installed in Hall A in February 2003. Both the systems have been continuously operating since then. The systematic block diagram of the linear raster system is described in Fig. 11.

The H-bridge power modules are located inside lead brick shielding with $2^{\prime \prime}$ wall thickness. The shielding house protects MOSFET elements from degradation of switching performance by longterm radiation exposure (the maximum local dose $\sim 200 \mathrm{rad}$ ). The lead shielding house has a good air-forced ventilation to keep the temperature of heat sink below $100 \mathrm{~K}$ in any case. Three parallel probes provide raster analog signals - the Pearson

Table 2

The overall performance of linear raster system

\begin{tabular}{ll}
\hline Type of magnet & Bedstead air-core \\
Beam energy $(\mathrm{GeV})$ & $0.8-12$ \\
Max. beam current $(\mu \mathrm{A})$ & 200 \\
Shape of raster trace & Triangular \\
Shape of raster boundaries & Rectangular \\
Max. rigidity $(\mathrm{GeV} / c)$ & $19.4 /\left(\Delta x_{1 / 2}(\mathrm{~mm})\right)$ \\
Max.bending power $(\mathrm{mrad})$ & $0.971 / \mathrm{p}(\mathrm{GeV} / \mathrm{c})$ \\
Max. magnet peak current $(\mathrm{A})$ & 100 \\
Raster frequency $(\mathrm{kHz})$ & $24.96 / 25.08$ \\
Frequency ratio & 1.00481 \\
Linear velocity $(\mathrm{m} / \mathrm{s})$ & 1000 \\
Turnig time at vertex $(\mathrm{ns})$ & 200 \\
Half-period/turnig time & 100 \\
Scan trace linearity $(\%)$ & 98 \\
Density uniformity $(\%)$ & 95 \\
Pattern stability & $2 \times 10^{-3}$ \\
Operational mode & Free run/external lock \\
Max. applied HV voltage $(\mathrm{V})$ & 500 \\
\hline
\end{tabular}

current probe for precision magnet current amplitude, field pickup coil $(d=1.5 \mathrm{~cm}, L=3.25 \mu \mathrm{H}$, 10 turns Awg 18 wire in a single layer, mounted at the center of the transverse plane of the raster magnet) for the field ramp signals calibrated by the Pearson output, and the LEM probe for DC offset detection to monitor the performance of $\mathrm{H}$-bridge HEXFETs during the operation.

Inside the power module, $\mathrm{HV}$ is applied by the UltraVolt high voltage unit, which is controlled by either a manual controller or by EPICS through HV I/O controller and RS232 connection. The EPICS keeps watch on HV voltage, consumed current, and DC offset of the bridge. The built-in H-bridge control waveform generator provides two sequences of driving signals to each shoulder of the H-bridge and a synchronization TTL signal to the experimental data aquisition system to identify the polarization of analog signals for instant beam position reconstruction.

The beam raster monitor VME module located inside experimental halls receives analog signals from the Pearson and field pickup, comparing the parameters between setting and readback to create fast shutdown detection for the machine safety operation. In the same location, a synchronized two-channel VME ADC is used to convert $x$ and $y$ analog signals to a $2 \mathrm{D}$ and $3 \mathrm{D}$ histogram on-line display for users and machine operators.

In the experimental counting house, the helicity synchronization unit receives helicity signals from the injector to generate two raster frequencies as described in Section 4.4. A local manual controller in the counting house gives users a parallel function of setting parameters in addition to the remote EPICS control if necessary.

$G_{0}$ experiment [8] studied target boiling phenomena at a fixed beam current $40 \mu \mathrm{A}$ beam [9] under the new raster system operation. The raster size was kept as $2 \times 2 \mathrm{~cm}^{2}$. They found that the target density fluctuation due to boiling of the liquid target material is as negligible as $40 \mathrm{ppm}$. If we compare the $40 \mathrm{ppm}$ density fluctuation of $G_{0}$ data with the data from the Hall C HMS luminosity scan in 2000 under the same condition but on operating the original Lissajous raster system, the relative yield drops down by $9.6 \%$ in $50 \mu \mathrm{A}$ beam current increment, an obvious 


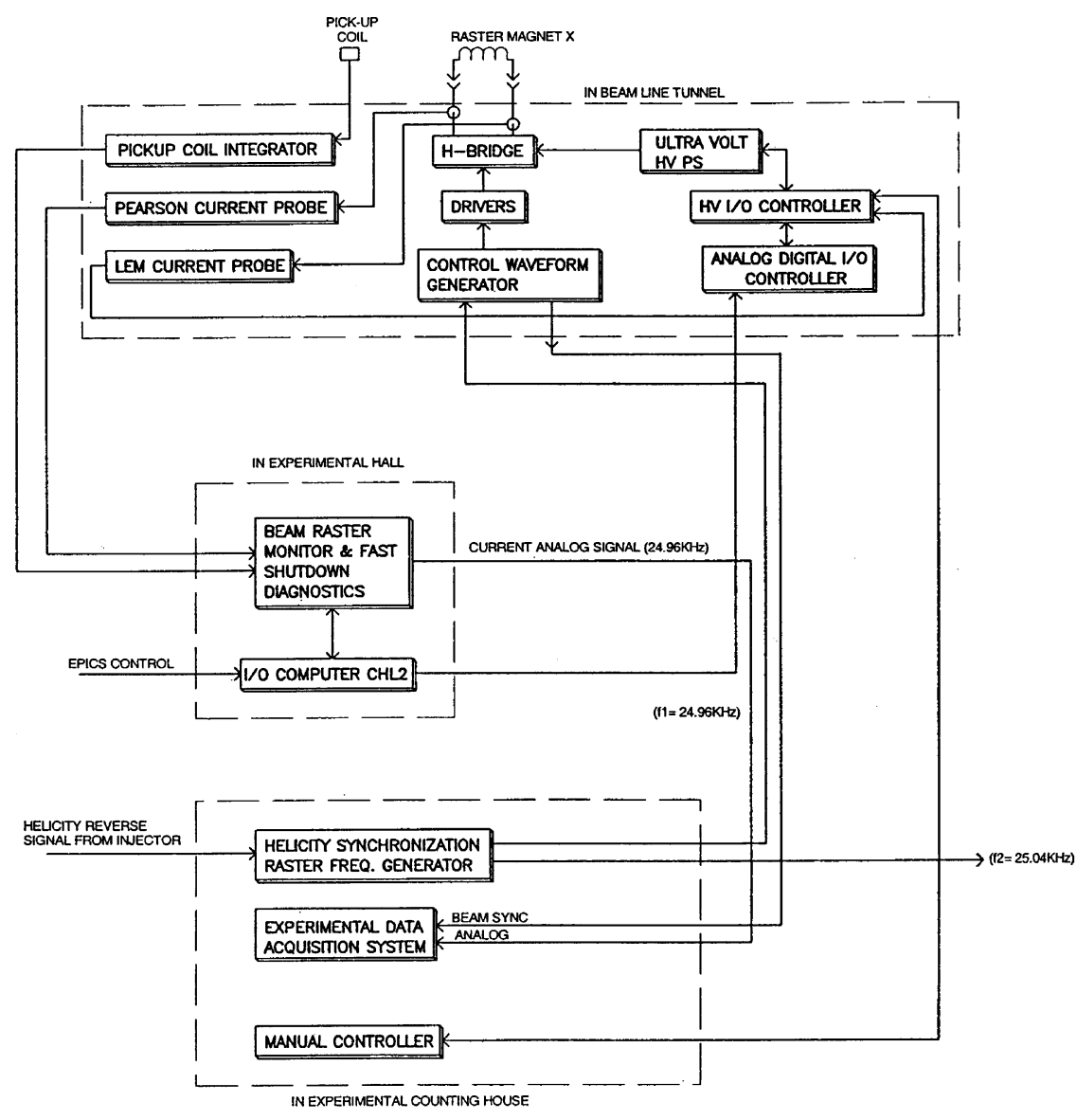

Fig. 11. The systematic block diagram of linear raster system for one magnet.

improvement of target heating effect by applying the new raster system can be addressed.

\section{Acknowledgements}

We take this opportunity to thank the prompt engineering and technical support from J. Dail and C. Apeldoorn of Accelerator Division Machine Shop for their hard work in the design and construction of H-bridge mechanics [10]. We also appreciate the importance of the skill and the effort of C. Cuevas and W. Gunning of Physics Electronics Group for the success of the design and a part of production of $\mathrm{H}$-Bridge driving $\mathrm{PC}$ boards and the application PIC-ADIO board as the interface of EPICS control and also the strong support from D. Wetherhold and C. Slominski of Accelerator Software Group to get the new system operation under the versatile EPICS control as well as the 2D/3D raster pattern on-line display.

This work was supported by the US Department of Energy, under Contract no. DE-AC0584ER40150.

\section{References}

[1] C. Yan, User's manual of Hall C target raster system, Internal Report, 1996.

[2] H. Bethe, in: S. Flugge (Ed.), Handbuch der Physik, vol. 24, Berlin, 1953, p. 273.

[3] F. Bloch, Ann. Phys. 16 (1933) 285. 
[4] F. Bloch, Z. Phys. B 1 (1933) 363.

[5] J.H. Mitchell, Global and local heating in the Hall C Cryo Targets, CEBAF Internal Report, March 2, 1993.

[6] J. Karn, Fast raster magnet measurement, Internal Report, September 30, 1996.

[7] C. Yan, et al., Nucl. Instr. and Meth. A 365 (1995) 261.
[8] D. Beck et al., Measurement of the Flavor Singlet form factor of the proton, PR91-017, 1992.

[9] Log entry no. 54917, Electronic Logbook of $G_{0}$ Experiment, 2002.

[10] N. Sinkine, C. Yan, C. Apeldoom, J. Dail, R. Wojcik, W. Gunning, Pending patent A linear raster magnet driver based on H-bridge technique, 2002. 